\title{
Engineering the Electronic Structure of NiFe Layered Double Hydroxide Nanosheet Array by Implanting Cationic Vacancies for Efficient Electrochemical Conversion of 5- Hydroxymethylfurfural to 2,5-
} Furandicarboxylic Acid

Yu-Feng Qi, ${ }^{a}$ Kai-Yao Wang ${ }^{a, b, c, *}$ Yan Sun, ${ }^{a}$ Jie Wang ${ }^{a}$ and Cheng Wang ${ }^{a, *}$

${ }^{a}$ Tianjin Key Laboratory of Advanced Functional Porous Materials, Institute for New Energy Materials and Low-Carbon Technologies, School of Materials Science and Engineering, Tianjin University of Technology, Tianjin 300384, P.R. China

${ }^{b}$ State Key Laboratory of Structural Chemistry, Fujian Institute of Research on the Structure of Matter, Chinese Academy of Sciences, Fuzhou, Fujian 350002, P.R. China

${ }^{c}$ National Engineering Research Center for Optoelectronic Crystalline Materials, Fuzhou, Fujian 350002, P.R. China

*Corresponding Authors: Kai-Yao Wang, Email: kaiyao0729@163.com; Cheng Wang, Email: cwang@tjut.edu.cn.

\section{Supporting Information Content:}

Number of pages: (S1-S17)

Number of Figures: 13 (Figure S1- Figure S13)

Number of Tables: 1 (Table S1) 


\section{Table of Contents}

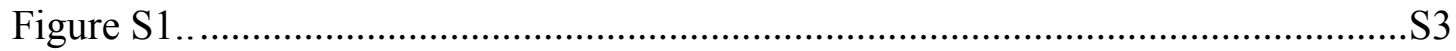

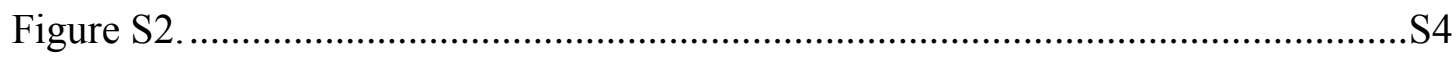

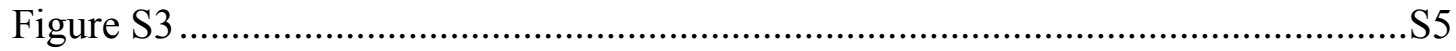

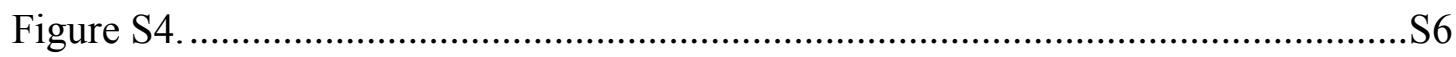

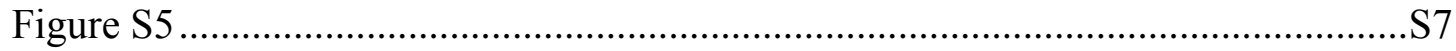

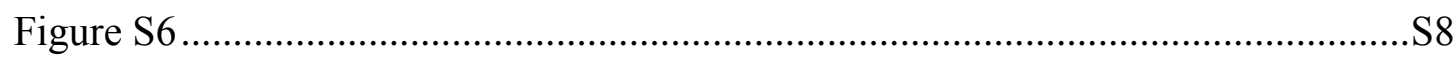

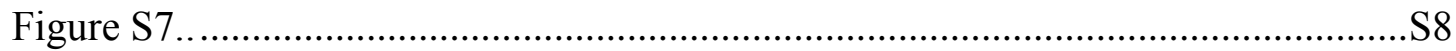

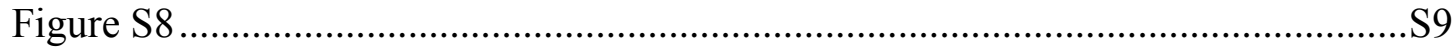

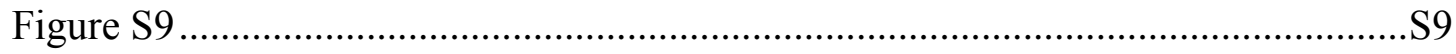

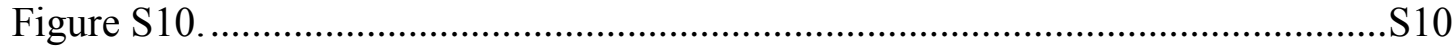

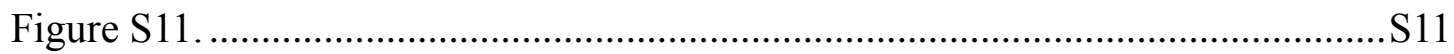

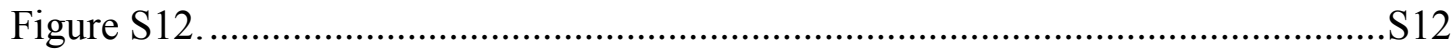

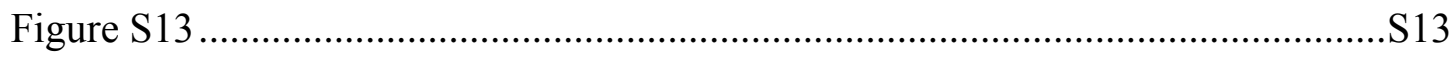

Table S1 . ................................................................................................

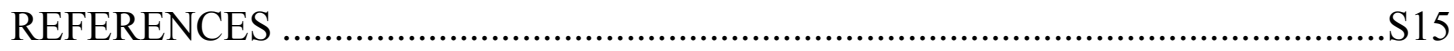



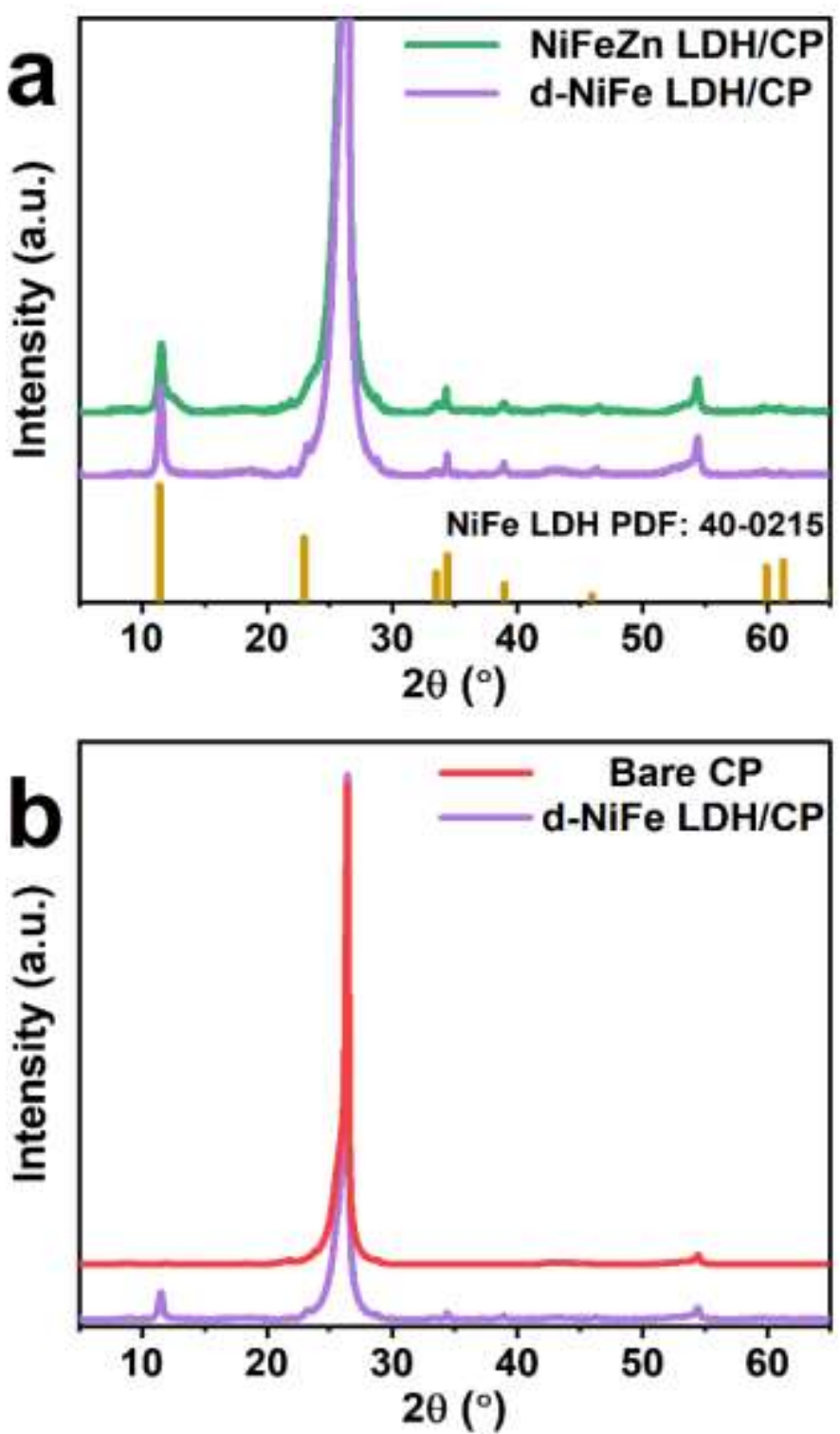

Figure S1. (a) XRD patterns of NiFeZn LDH/CP and d-NiFe LDH/CP. (b) XRD patterns of bare carbon paper (CP) and d-NiFe LDH/CP. 

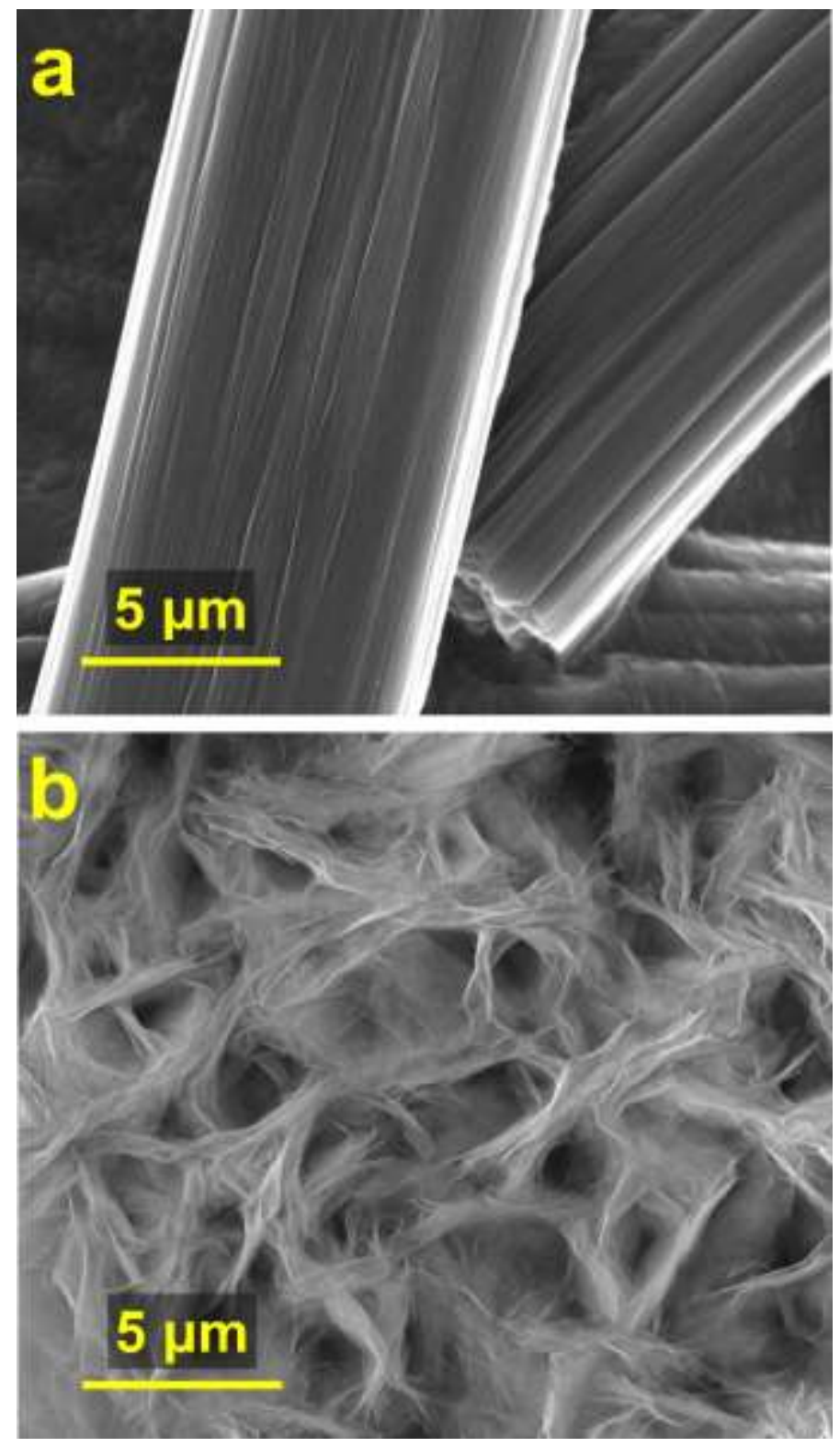

Figure S2. SEM images of (a) bare CP and (b) NiFeZn LDH/CP. 

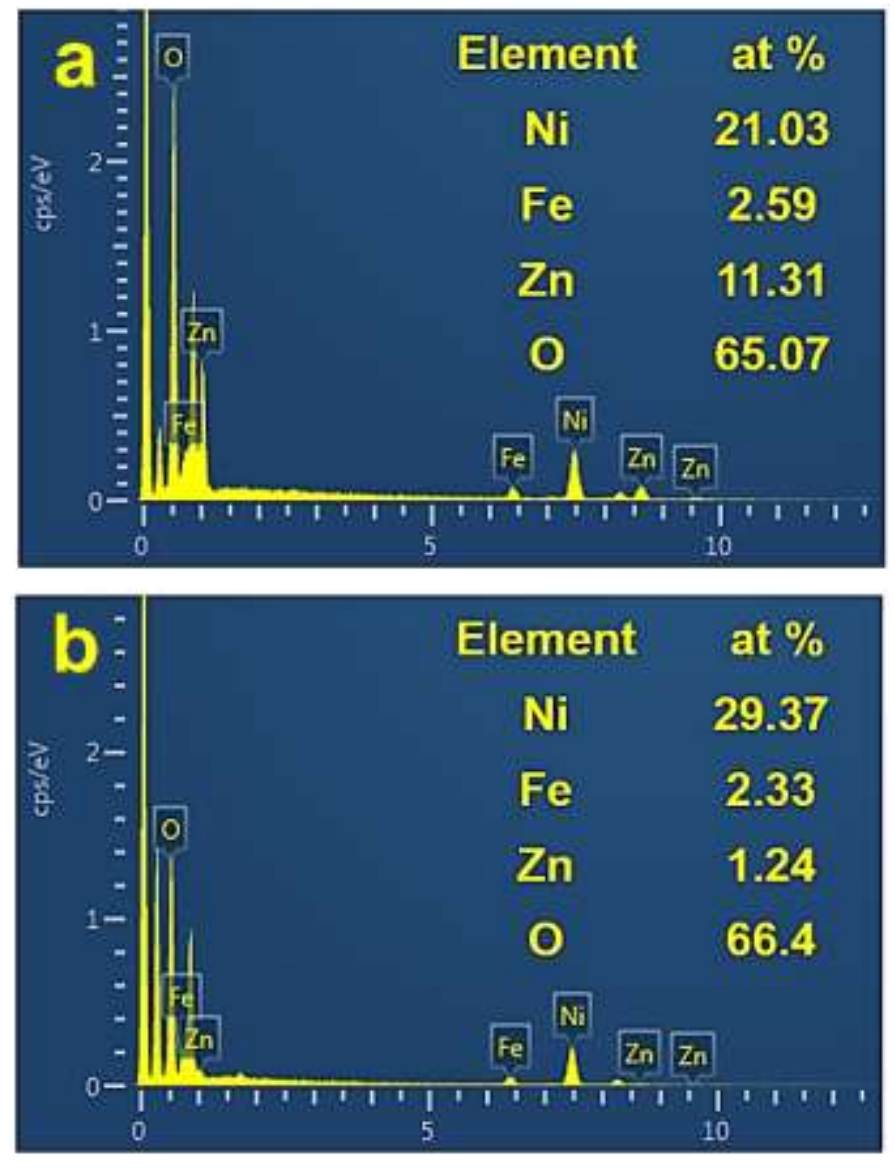

Figure S3. EDX spectra of (a) NiFeZn LDH/CP and (b) d-NiFe LDH/CP. 

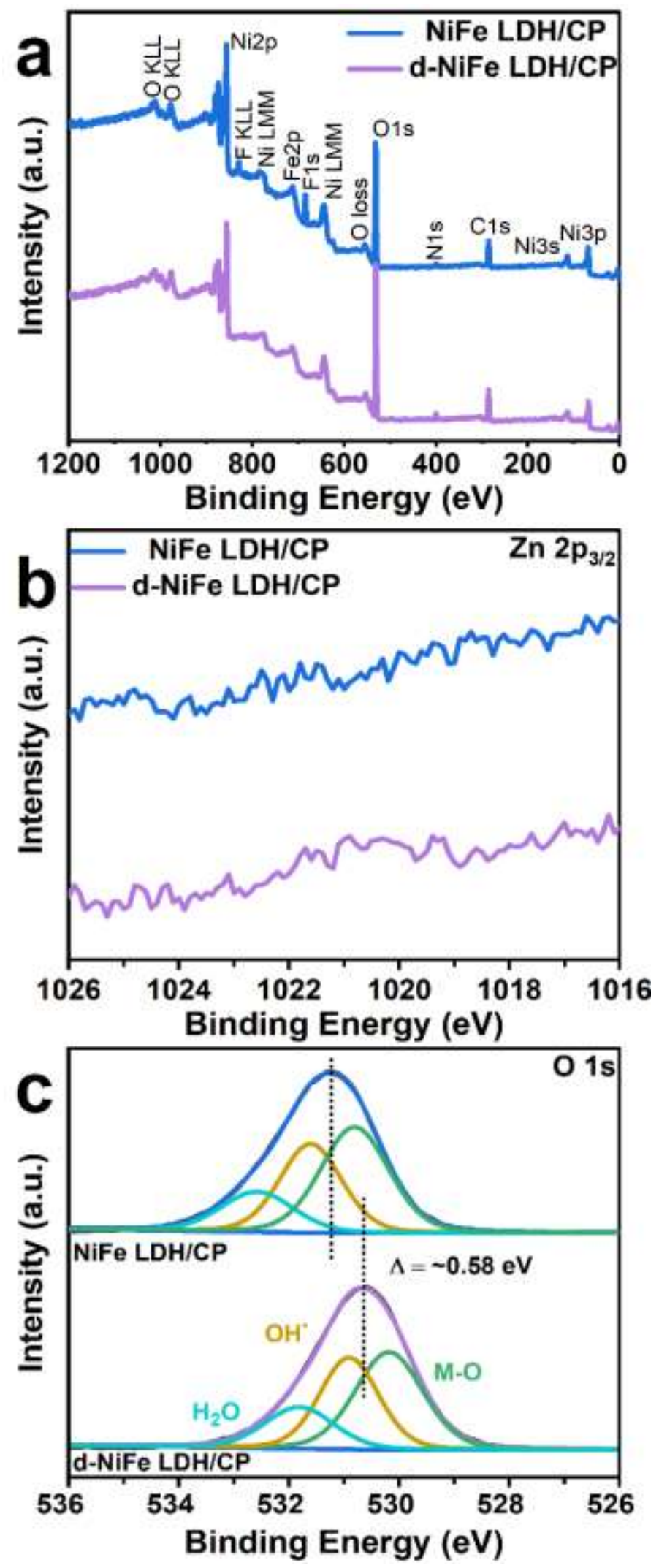

Figure S4. (a) XPS overall spectra of NiFe LDH/CP and d-NiFe LDH. (b,c) Highresolution spectra of $\mathrm{Zn} 2 \mathrm{p}$ and $\mathrm{O} 1 \mathrm{~s}$ regions for $\mathrm{NiFe} \mathrm{LDH} / \mathrm{CP}$ and $\mathrm{d}-\mathrm{NiFe} \mathrm{LDH} / \mathrm{CP}$. 

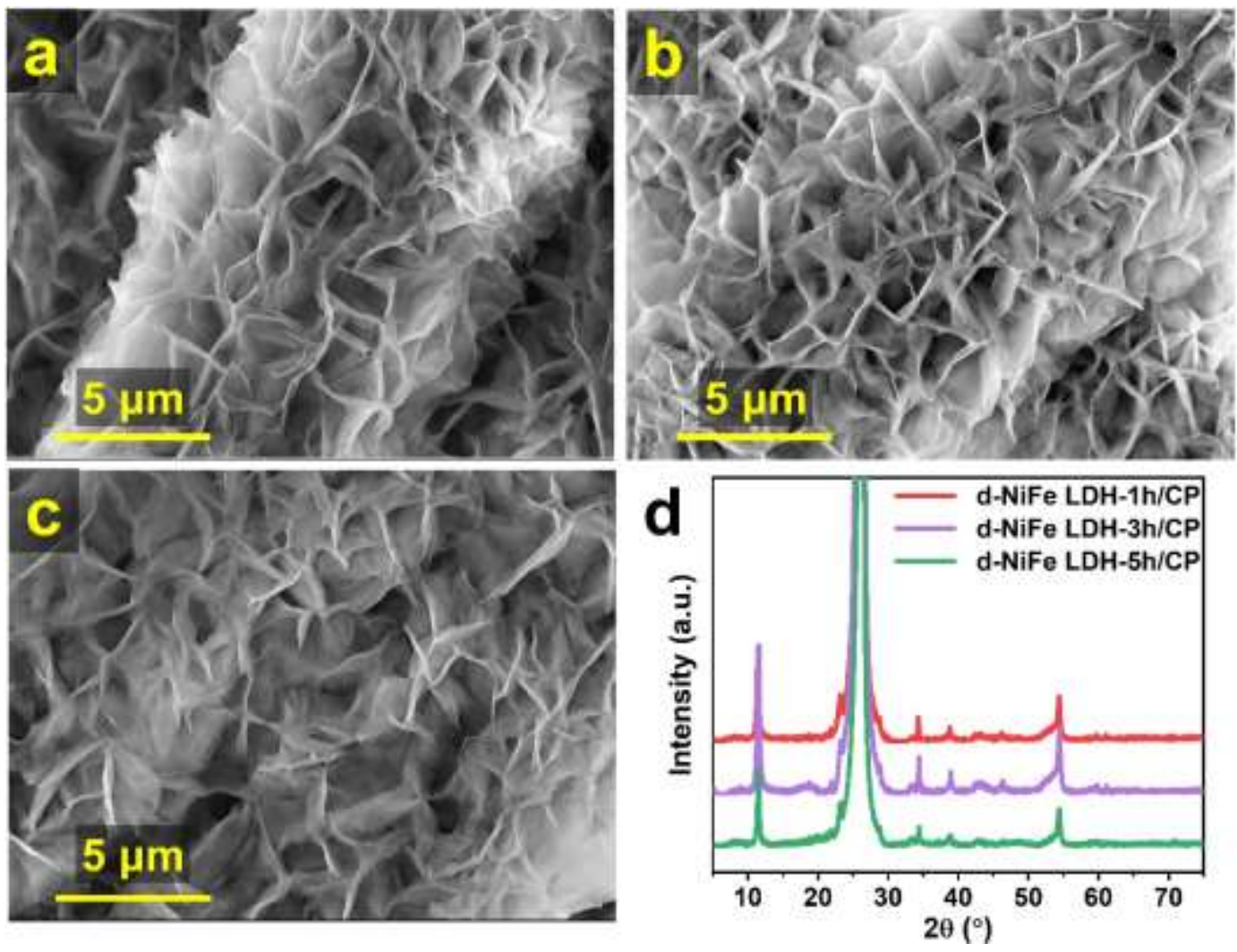

Figure S5. SEM images for d-NiFe LDH/CP with different etching time of (a) $1 \mathrm{~h}$, (b)

3h and (c) 5h. (d) XRD spectra of corresponding d-NiFe LDH/CP with different etching durations. 

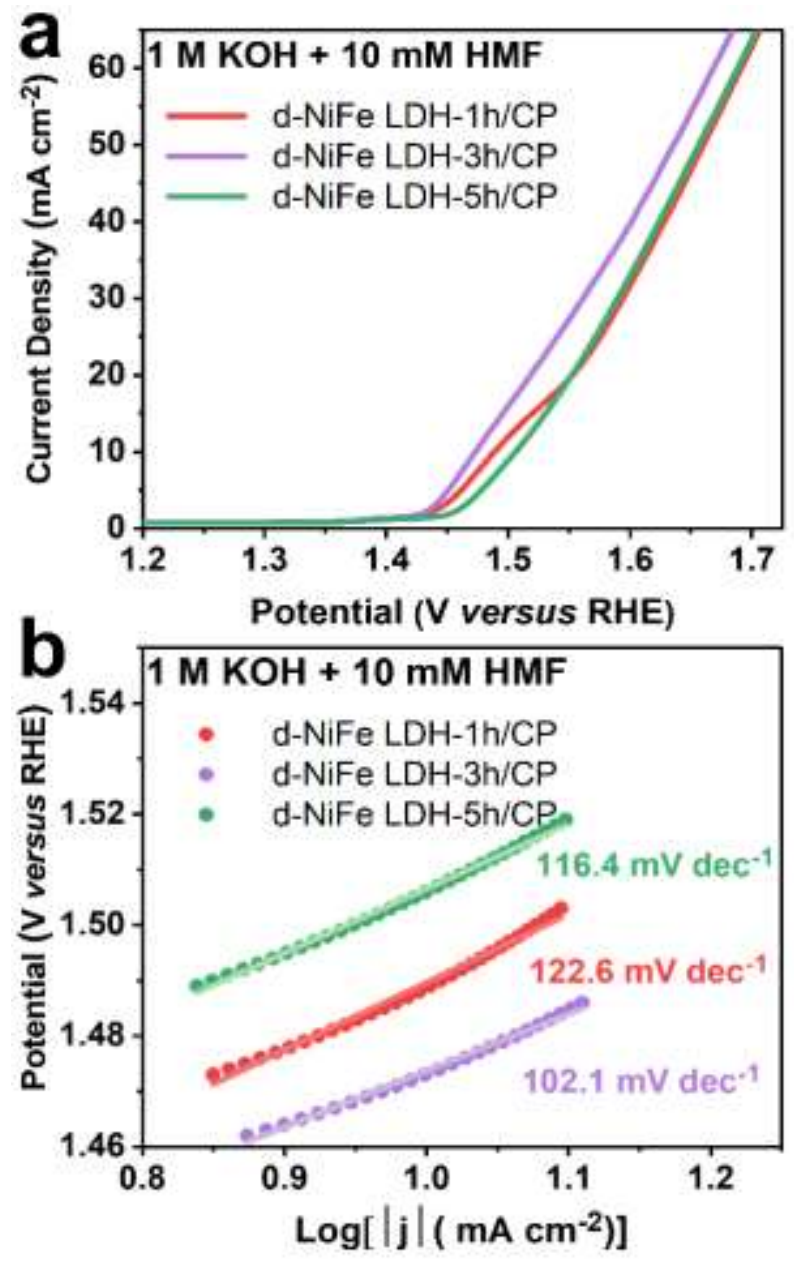

Figure S6. (a) LSV curves and (b) corresponding Tafel plots of d-NiFe LDH/CP with different etching times.

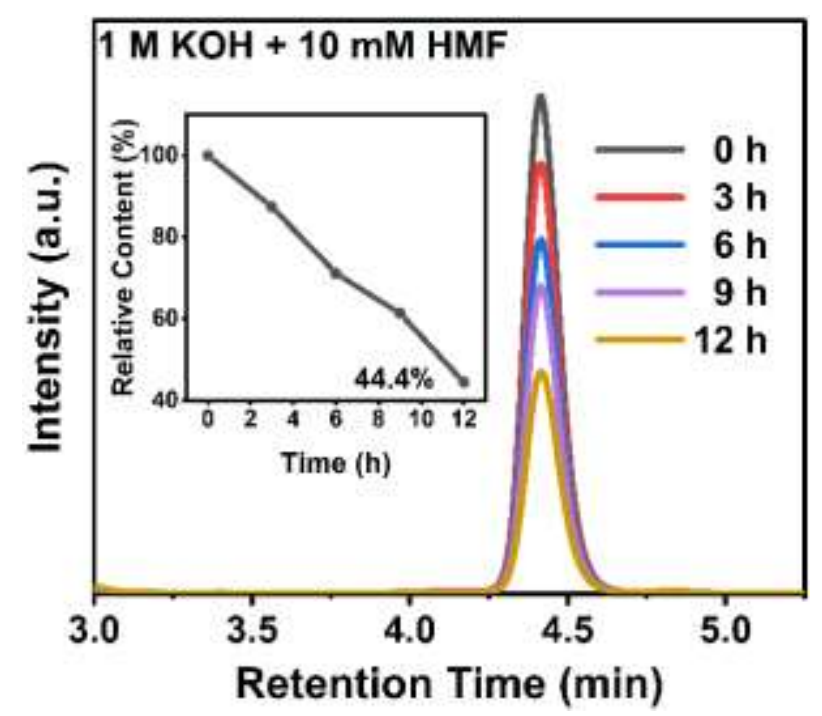

Figure S7. Stability test of $10 \mathrm{mM}$ HMF in $1 \mathrm{M} \mathrm{KOH}$ aqueous solution under room temperature without stirring. 


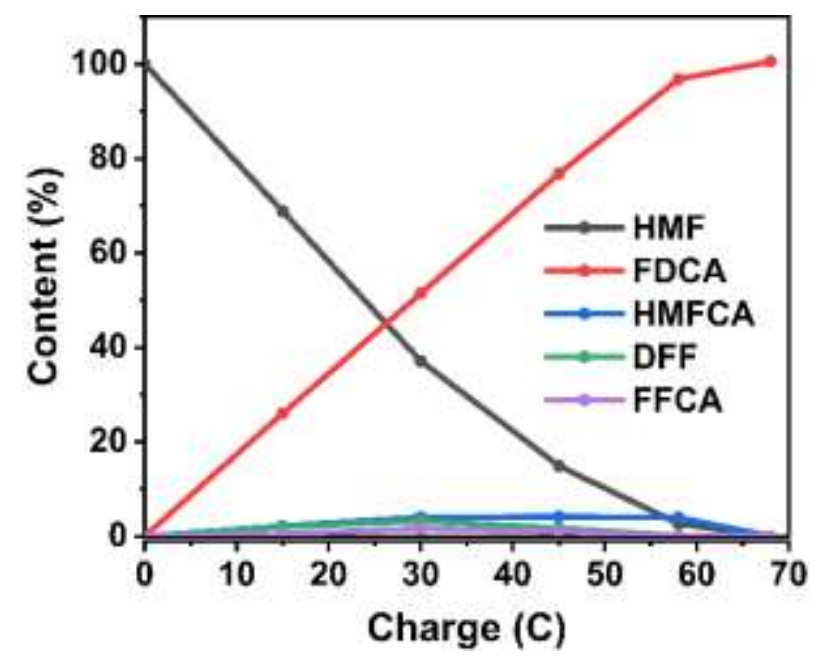

Figure S8. Plots of contents for HMF, HMFCA, FFCA, DFF and FDCA versus charge.

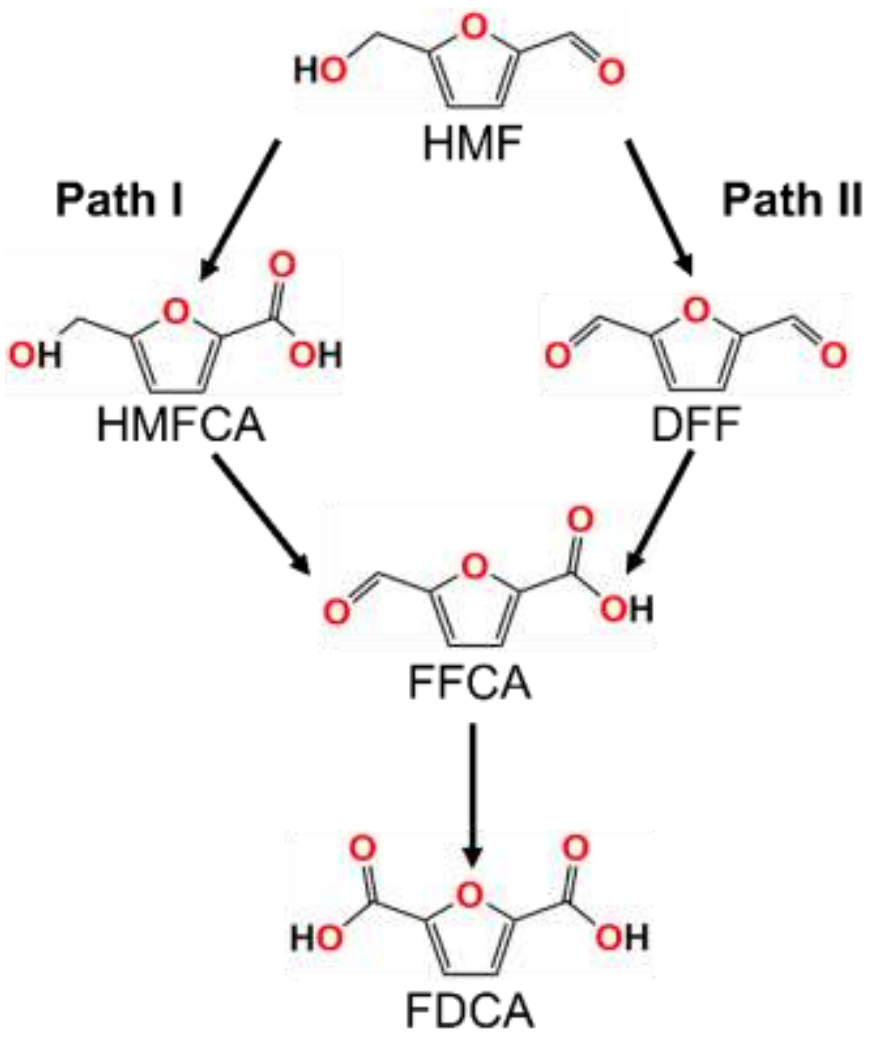

Figure S9. Two possible pathways for HMF oxidation. 


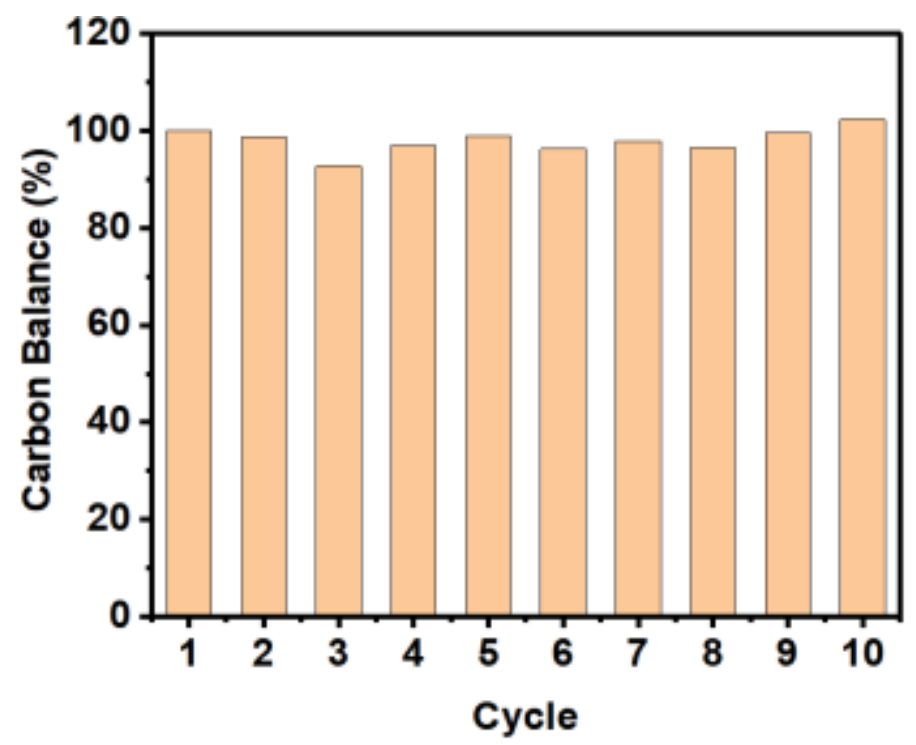

Figure S10. Calculated carbon balance during the stability test cycles. 

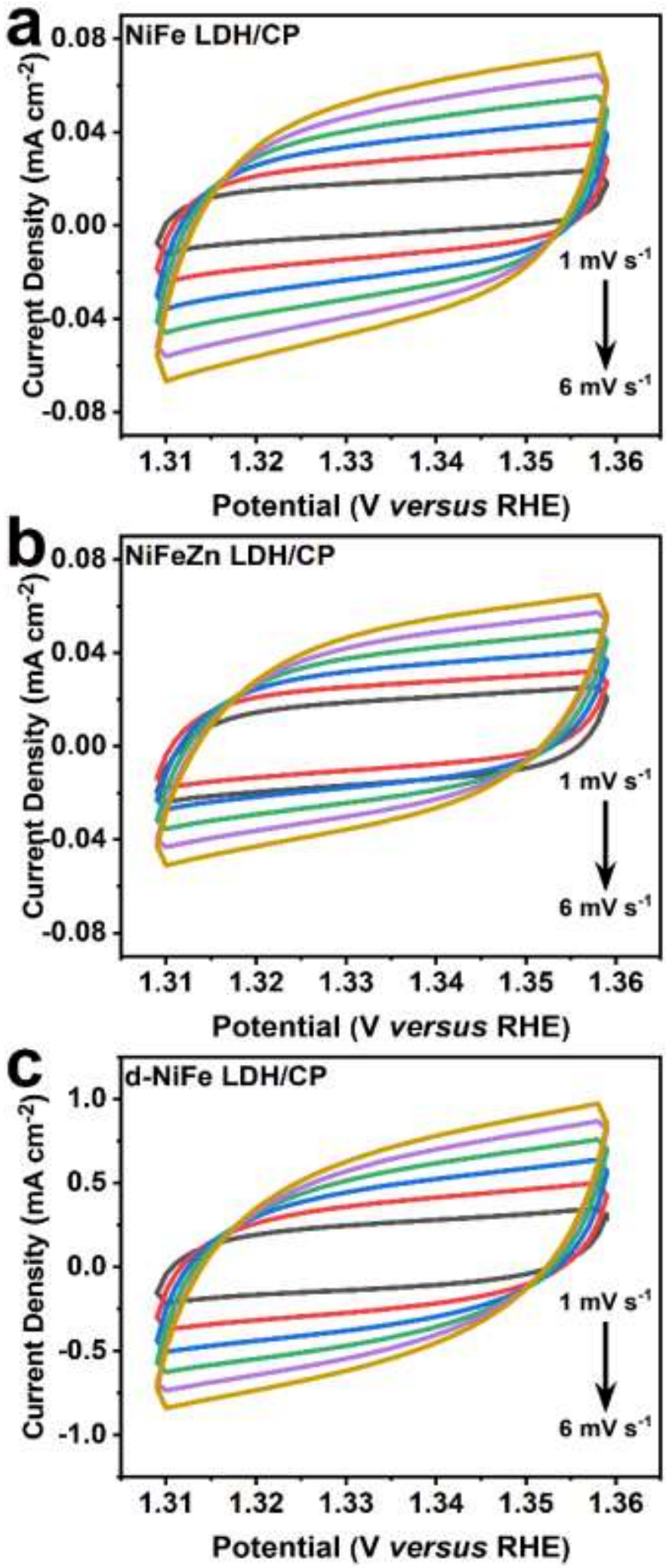

Figure S11. CV curves of (a) $\mathrm{NiFe} \mathrm{LDH} / \mathrm{CP}$, (b) $\mathrm{NiFeZn} \mathrm{LDH/CP}$ and (c) d-NiFe $\mathrm{LDH} / \mathrm{CP}$ with scan rates of $1,2,3,4,5$ and $6 \mathrm{mV} \mathrm{s}^{-1}$ at potential window from 1.31$1.36 \mathrm{~V}$. 


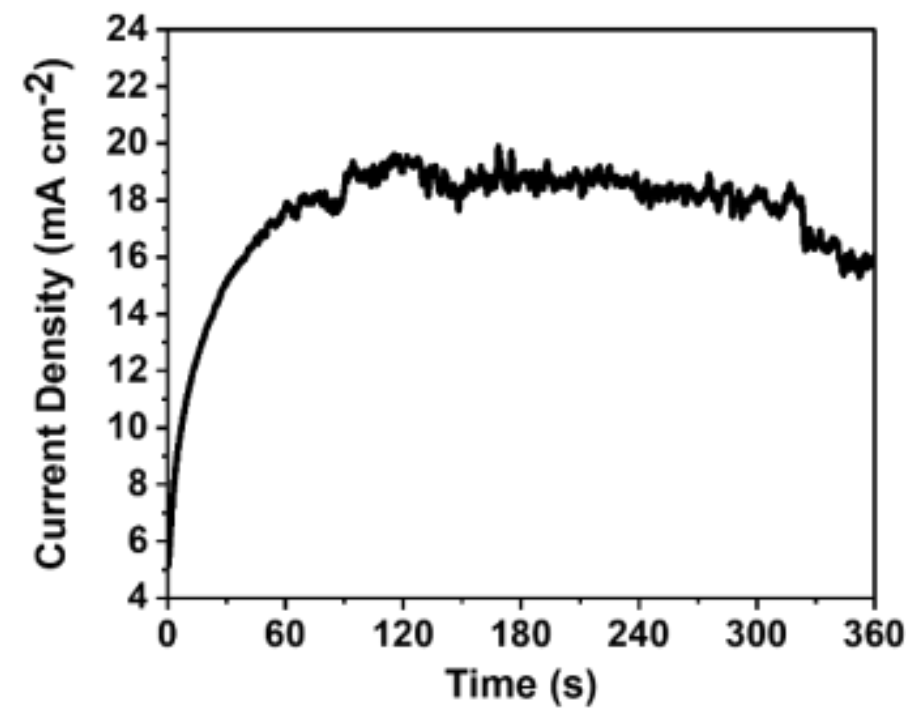

Figure S12. Early stage of i-t curve for electro-oxidation of HMF on d-NiFe LDH/CP electrode. 

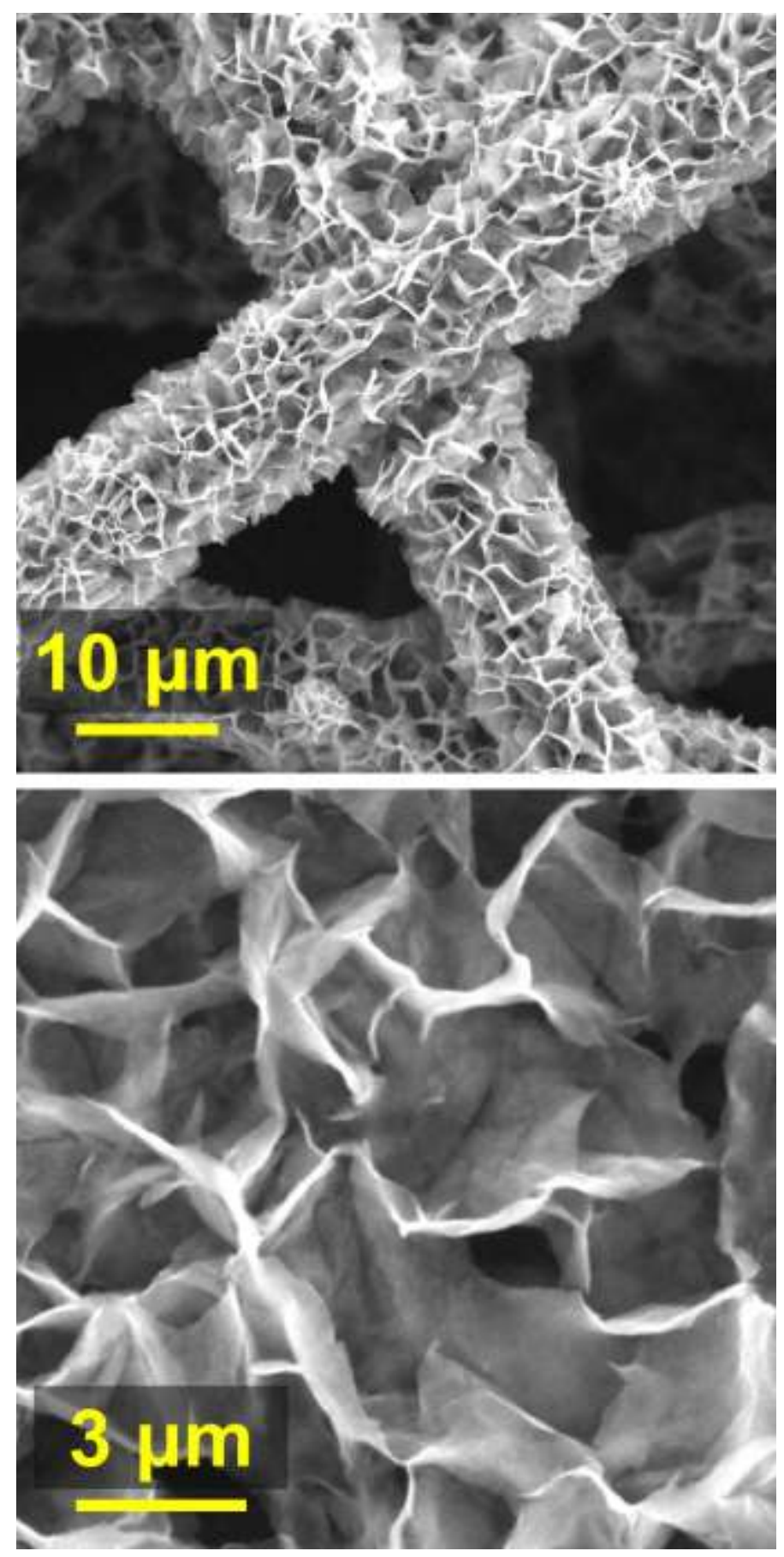

Figure S13. SEM images of d-NiFe $\mathrm{LDH} / \mathrm{CP}$ after electrocatalytic process with different magnifications. 
Table S1. Catalytic performance comparison of the d-NiFe LDH/CP and other reported catalysts toward HMF electrocatalytic oxidation in alkaline medium.

\begin{tabular}{|c|c|c|c|c|}
\hline Catalyst & $\begin{array}{c}\text { HMF Conversion } \\
(\%)\end{array}$ & $\begin{array}{c}\text { FDCA Yield } \\
(\%)\end{array}$ & $\begin{array}{c}\text { Faradic Efficiency } \\
(\%)\end{array}$ & Ref. \\
\hline $\mathrm{d}-\mathrm{NiFe} \mathrm{LDH} / \mathrm{CP}(\mathrm{Q}=58 \mathrm{C})$ & 97.3 & 96.8 & 84.5 & This work \\
\hline $\mathrm{d}-\mathrm{NiFe} \mathrm{LDH} / \mathrm{CP}(\mathrm{Q}=68 \mathrm{C})$ & 100 & 100 & 74.8 & This work \\
\hline $\mathrm{BNC}$ & 71 & 57 & 57 & 1 \\
\hline $\mathrm{N}-\mathrm{MoO}_{2} / \mathrm{Ni}_{3} \mathrm{~S}_{2} \mathrm{NF}$ & 90 & $\sim 90$ & / & 2 \\
\hline NiCoFe-LDHs & $95.5\left(55^{\circ} \mathrm{C}\right)$ & 81 & $\sim 90$ & 3 \\
\hline $\mathrm{Co}-\mathrm{P} / \mathrm{CF}$ & 100 & 90 & / & 4 \\
\hline $\mathrm{VN} / \mathrm{NF}$ & 98 & 96 & 84 & 5 \\
\hline NiO-CMK-1 & 65 & 51 & 70 & 6 \\
\hline $\mathrm{NiCo}_{2} \mathrm{O}_{4} / \mathrm{NF}$ & 99.6 & 90.4 & 90.8 & 7 \\
\hline $\mathrm{Cu}_{1.8} \mathrm{Co}_{0.2} \mathrm{PO}_{4} \mathrm{~F}$ & 89.5 & 85 & 95 & 8 \\
\hline $\mathrm{CoNW} / \mathrm{NF}$ & 100 & 96.8 & 96.6 & 9 \\
\hline $\mathrm{CoO}-\mathrm{CoSe}_{2}$ & 100 & 99 & 97.9 & 10 \\
\hline $\mathrm{NiB}_{\mathrm{x}}-\mathrm{P}_{\mathrm{y}}$ & 99.9 & 90.6 & 92.5 & 11 \\
\hline Co-doped NiBDC-NF & 70 & 99 & 78.8 & 12 \\
\hline $\mathrm{Ni}_{0.9}-\mathrm{Cu}_{0.1}(\mathrm{OH})_{2}$ & 100 & 91.2 & 91.2 & 13 \\
\hline $\mathrm{NiO}-\mathrm{Co}_{3} \mathrm{O}_{4}$ & $\sim 100$ & 98 & 96 & 14 \\
\hline NiCoMn-LDHs/NF & $100\left(35^{\circ} \mathrm{C}\right)$ & 91.7 & 65 & 15 \\
\hline $\mathrm{WO}_{3} / \mathrm{Ni}$ & 99.4 & 88.3 & 88 & 16 \\
\hline $\mathrm{NiCO}_{2} \mathrm{O}_{4}-\mathrm{CFP}$ & 98.4 & 94.3 & 89.6 & 17 \\
\hline PBA-CoCo & $\sim 90$ & $\sim 85$ & 62 & 18 \\
\hline
\end{tabular}




\section{REFERENCES}

1. Qin, Q.; Heil, T.; Schmidt, J.; Schmallegger, M.; Gescheidt, G.; Antonietti, M.; Oschatz, M., Electrochemical Fixation of Nitrogen and Its Coupling with Biomass Valorization with a Strongly Adsorbing and Defect Optimized Boron-Carbon-Nitrogen Catalyst. ACS Appl. Energy Mater. 2019, 2 (11), 8359-8365, DOI 10.1021/acsaem.9b01852.

2. Wang, L.; Cao, J.; Lei, C.; Dai, Q.; Yang, B.; Li, Z.; Zhang, X.; Yuan, C.; Lei, L.; Hou, Y., Strongly Coupled 3D N-Doped $\mathrm{MoO}_{2} / \mathrm{Ni}_{3} \mathrm{~S}_{2}$ Hybrid for High Current Density Hydrogen Evolution Electrocatalysis and Biomass Upgrading. ACS Appl. Mater. Interfaces 2019, 11 (31), 27743-27750, DOI 10.1021/acsami.9b06502.

3. Zhang, M.; Liu, Y.; Liu, B.; Chen, Z.; Xu, H.; Yan, K., Trimetallic NiCoFe-Layered Double Hydroxides Nanosheets Efficient for Oxygen Evolution and Highly Selective Oxidation of Biomass-Derived 5-Hydroxymethylfurfural. ACS Catal. 2020, 10 (9), 5179-5189, DOI 10.1021/acscatal.0c00007.

4. Jiang, N.; You, B.; Boonstra, R.; Terrero Rodriguez, I. M.; Sun, Y., Integrating Electrocatalytic 5-Hydroxymethylfurfural Oxidation and Hydrogen Production via Co-P-Derived Electrocatalysts. ACS Energy Lett. 2016, 1 (2), 386-390, DOI 10.1021/acsenergylett.6b00214.

5. Li, S.; Sun, X.; Yao, Z.; Zhong, X.; Cao, Y.; Liang, Y.; Wei, Z.; Deng, S.; Zhuang, G.; Li, X.; Wang, J., Biomass Valorization via Paired Electrosynthesis Over Vanadium Nitride $\square$ Based Electrocatalysts. Adv. Funct. Mater. 2019, 29 (42), 1904780, DOI 10.1002/adfm.201904780.

6. Holzhäuser, F. J.; Janke, T.; Öztas, F.; Broicher, C.; Palkovits, R., Electrocatalytic Oxidation of $5 \square$ Hydroxymethylfurfural into the Monomer 2,5 $\square$ Furandicarboxylic Acid using Mesostructured Nickel Oxide. Adv. Sustainable Syst. 2020, 4 (10). 1900151, DOI 10.1002/adsu.201900151. 
7. Kang, M. J.; Park, H.; Jegal, J.; Hwang, S. Y.; Kang, Y. S.; Cha, H. G., Electrocatalysis of 5hydroxymethylfurfural at cobalt based spinel catalysts with filamentous nanoarchitecture in alkaline media. Appl. Catal. B 2019, 242, 85-91, DOI 10.1016/j.apcatb.2018.09.087.

8. Lemoine, K.; Inaguma, Y.; Heidary, N.; Kornienko, N., Mechanochemical synthesis of cobalt/copper fluorophosphate generates a multifunctional electrocatalyst. Chem. Commun. 2020, 56 (65), 9276-9279, DOI 10.1039/d0cc02815k.

9. Zhou, Z.; Chen, C.; Gao, M.; Xia, B.; Zhang, J., In situ anchoring of a $\mathrm{Co}_{3} \mathrm{O}_{4}$ nanowire on nickel foam: an outstanding bifunctional catalyst for energy-saving simultaneous reactions. Green Chem. 2019, 21 (24), 6699-6706, DOI 10.1039/c9gc02880c.

10. Huang, X.; Song, J.; Hua, M.; Xie, Z.; Liu, S.; Wu, T.; Yang, G.; Han, B., Enhancing the electrocatalytic activity of $\mathrm{CoO}$ for the oxidation of 5-hydroxymethylfurfural by introducing oxygen vacancies. Green Chem. 2020, 22 (3), 843-849, DOI 10.1039/c9gc03698a.

11. Song, X.; Liu, X.; Wang, H.; Guo, Y.; Wang, Y., Improved Performance of Nickel Boride by Phosphorus Doping as an Efficient Electrocatalyst for the Oxidation of 5-Hydroxymethylfurfural to 2,5-Furandicarboxylic Acid. Ind. Eng. Chem. Res. 2020, 59 (39), 17348-17356, DOI 10.1021/acs.iecr.0c01312.

12. Cai, M.; Zhang, Y.; Zhao, Y.; Liu, Q.; Li, Y.; Li, G., Two-dimensional metal-organic framework nanosheets for highly efficient electrocatalytic biomass 5-(hydroxymethyl)furfural (HMF) valorization. J. Mater. Chem. A 2020, 8 (39), 20386-20392, DOI 10.1039/d0ta07793c.

13. Zhang, J.; Yu, P.; Zeng, G.; Bao, F.; Yuan, Y.; Huang, H., Boosting HMF oxidation performance via decorating ultrathin nickel hydroxide nanosheets with amorphous copper hydroxide islands. $J$. Mater. Chem. A 2021, 9 (15), 9685-9691, DOI 10.1039/d0ta11678e. 
14. Lu, Y.; Dong, C.-L.; Huang, Y.-C.; Zou, Y.; Liu, Y.; Li, Y.; Zhang, N.; Chen, W.; Zhou, L.; Lin, H.; Wang, S., Hierarchically nanostructured $\mathrm{NiO}-\mathrm{Co}_{3} \mathrm{O}_{4}$ with rich interface defects for the electrooxidation of 5-hydroxymethylfurfural. Sci. China Chem. 2020, 63 (7), 980-986, DOI $10.1007 / \mathrm{s} 11426-020-9749-8$.

15. Liu, B.; Xu, S.; Zhang, M.; Li, X.; Decarolis, D.; Liu, Y.; Wang, Y.; Gibson, E. K.; Catlow, C. R. A.; Yan, K., Electrochemical upgrading of biomass-derived 5-hydroxymethylfurfural and furfural over oxygen vacancy-rich NiCoMn-layered double hydroxides nanosheets. Green Chem. 2021, 23 (11), 4034-4043, DOI 10.1039/d1gc00901j.

16. Hu, K.; Zhang, M.; Liu, B.; Yang, Z.; Li, R.; Yan, K., Efficient electrochemical oxidation of 5hydroxymethylfurfural to 2,5-furandicarboxylic acid using the facilely synthesized 3D porous $\mathrm{WO}_{3} / \mathrm{Ni}$ electrode. Mol. Catal. 2021, 504. 111459, DOI 10.1016/j.mcat.2021.111459.

17. Zhong, Y.; Ren, R.-Q.; Qin, L.; Wang, J.-B.; Peng, Y.-Y.; Li, Q.; Fan, Y.-M., Electrodeposition of hybrid nanosheet-structured $\mathrm{NiCo}_{2} \mathrm{O}_{4}$ on carbon fiber paper as a non-noble electrocatalyst for efficient electrooxidation of 5-hydroxymethylfurfural to 2,5-furandicarboxylic acid. New J. Chem. 2021, 45 (25), 11213-11221, DOI 10.1039/d1nj01489g.

18. Deng, C.; Wu, K. H.; Lu, X.; Cheong, S.; Tilley, R. D.; Chiang, C. L.; Lin, Y. C.; Lin, Y. G.; Yan, W.; Scott, J.; Amal, R.; Wang, D. W., Ligand-Promoted Cooperative Electrochemical Oxidation of Bio-Alcohol on Distorted Cobalt Hydroxides for Bio-Hydrogen Extraction. ChemSusChem 2021, 14 (12), 2612-2620, DOI 10.1002/cssc.202100722. 